\title{
Silver Core-Shell Nanoclusters Exhibiting Strong Growth Inhibition of Plant-Pathogenic Fungi
}

\author{
Viet Anh Ho, ${ }^{1}$ Phuong Thu Le, ${ }^{2}$ Thi Phuong Nguyen, ${ }^{1}$ Cuu Khoa Nguyen, ${ }^{1}$ \\ Vinh Truong Nguyen, ${ }^{3}$ and Ngoc Quyen Tran ${ }^{1}$ \\ ${ }^{1}$ Department of Materials and Pharmaceutical Chemistry, Institute of Applied Materials Science, \\ Vietnam Academy of Science and Technology, Ho Chi Minh City 700000, Vietnam \\ ${ }^{2}$ University of Science and Technology of Hanoi, Hanoi 100000, Vietnam \\ ${ }^{3}$ School of Agriculture and Forestry, Hue University, Hue 530000, Vietnam
}

Correspondence should be addressed to Ngoc Quyen Tran; tnquyen@iams.vast.vn

Received 17 November 2014; Accepted 19 January 2015

Academic Editor: Antonios Kelarakis

Copyright (c) 2015 Viet Anh Ho et al. This is an open access article distributed under the Creative Commons Attribution License, which permits unrestricted use, distribution, and reproduction in any medium, provided the original work is properly cited.

\begin{abstract}
We introduced a novel method to prepare silver core-shell nanoclusters (NCs) in which 3,4-dihydroxyphenyl acetic-conjugated oligochitosan (DHPAC) reduced silver salt and subsequently protected the produced nanosilver via mussel adhesion mechanism. Results indicated that the degree of conjugation was 14 dihydroxyphenyl acetamide moieties over 100 glucosamine units of oligochitosan. We used chitosan-catechol derivative to prepare the well-defined silver core-shell NCs and applied UV-visible spectroscopy, transmission electron microscopy (TEM), and X-ray diffraction (XRD) techniques to characterize the NCs. The core-shell NCs exhibited strong growth inhibition of plant-pathogenic fungi such as Phytophthora capsici, Phytophthora nicotianae, and Phytophthora colocasiae. These positive results may offer great potential to produce silver core-DHPAC shell NCs for several biomedical applications.
\end{abstract}

\section{Introduction}

Usage of metallic nanoparticles (NPs) for biomedical and industrial applications has recently had much attention due to several novel properties, such as optical, catalytic, and antimicrobial properties, compared to their bulk metallic forms [13]. Several kinds of metallic nanoparticles like silver, copper, and gold exhibit activity against some microbes, pathogenic fungi, and microorganisms [4-8]. Among them, silver NPs also had strong activity against various plant-pathogenic fungi, such as Phytophthora and Corticium fungi [9-12]. This is the reason why industrial and agricultural fields have studied silver nanoparticles (AgNPs) for their antibacterial or fungicide applications.

In tropical countries, Phytophthora has caused much damage to agriculture, especially economic plants. The Phytophthora $(P$.$) fungi caused serious damage to durian tree$ (P. palmivora), black pepper/chili/tomato plant (P. capsici), tobacco, citrus (P. nicotianae), and taro (P. colocasiae) which resulted in dead plants and significant decrease in productivity $[13,14]$. The plant-pathogenic fungi are controlled mainly by treatment with chemical fungicides, such as fosetylaluminium, metalaxyl and potassium phosphonate; however, results showed that these fungicides exhibited a low economic efficacy due to high cost, toxicity to humans, and environment pollution, when used in large scale. For these reasons, finding an environment-friendly antimicrobial agent against pathogenic fungi is important in the sustainable agriculture development. This is why AgNPs-based antimicrobial agents for agricultural field have recently received much attention, especially when the AgNPs can be prepared by simple methods and without using toxic reducing agents $[10,12]$.

In this study, we introduced a simple method to prepare silver (Ag) core-shell nanoclusters (NCs) in which DHPAC reduced silver salt and subsequently protected the produced AgNPs via its surface adhesion with 3,4-dihydroxyphenyl acetamide moieties (demonstrated in Figure 1). This is 




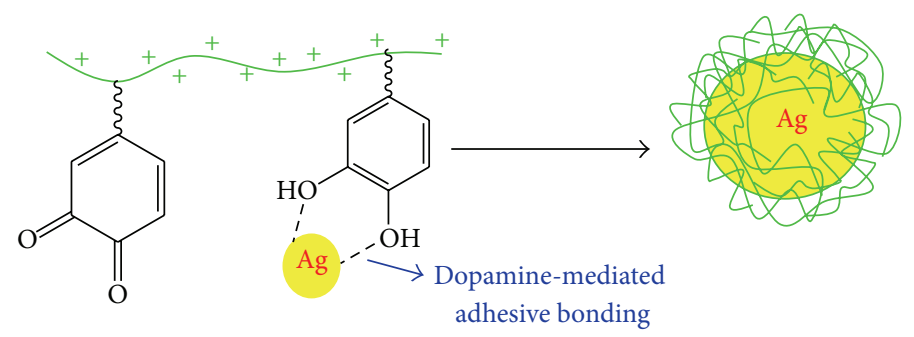

FIgURE 1: Preparation of Ag core-DHPAC shell via mussel adhesion mechanism.

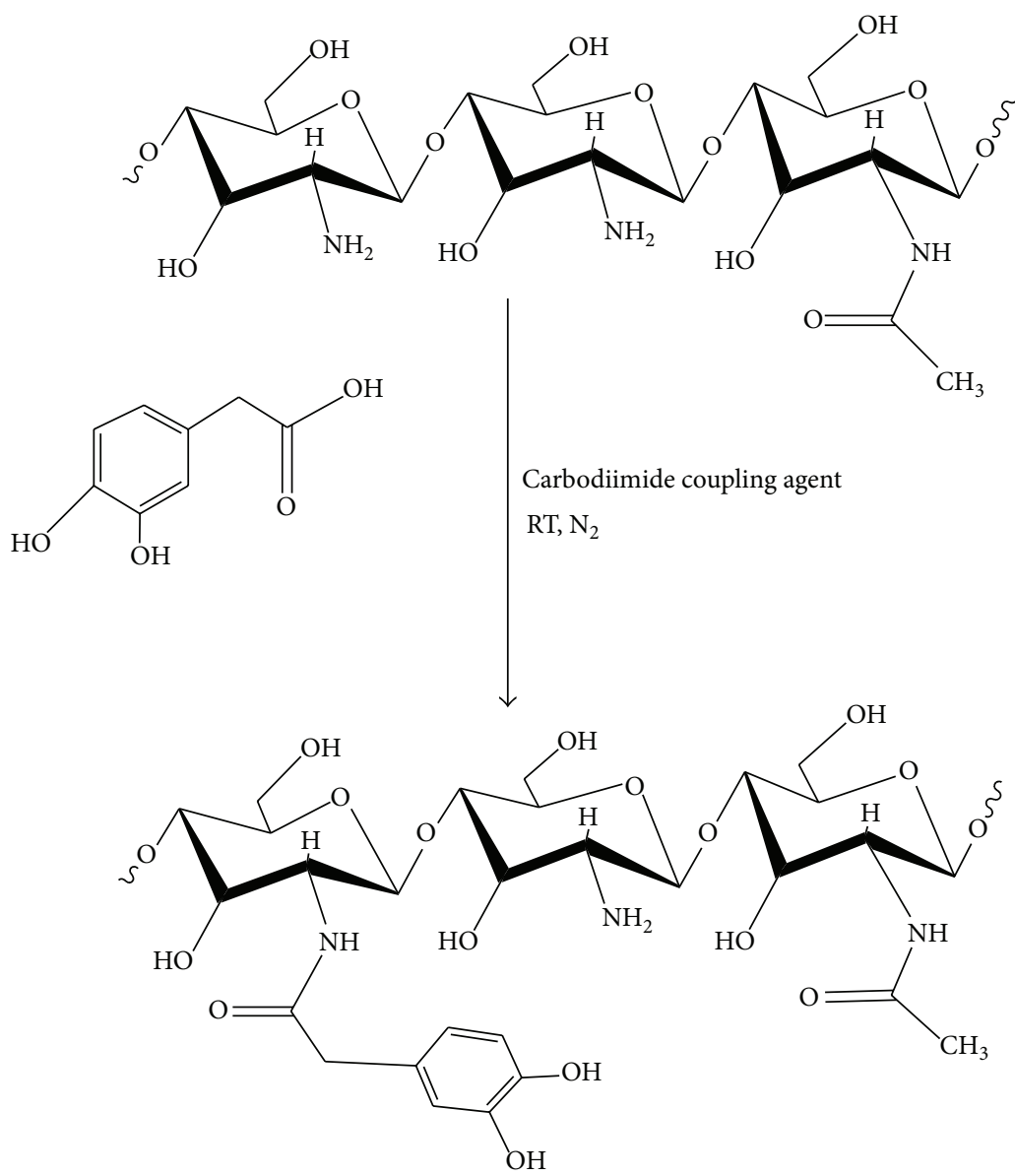

SCHEME 1: Synthetic route of DHPAC.

a biomimetic adhesion mechanism like mussel adhesive proteins $[15,16]$. The Ag core-shell NCs were characterized by UV-Vis spectroscopy, TEM, and XRD techniques. Their activities against plant-pathogenic fungi were evaluated on $P$. capsici, P. nicotianae, and P. colocasiae.

\section{Experimental}

2.1. Materials. Silver nitrate $\left(\mathrm{AgNO}_{3}\right)$ and 3,4-dihydroxyphenyl acetic acid (DHPA) were purchased from Acros Organics (Belgium). Oligochitosan (45,000 D, 85\% deacetylation) was prepared in the Department of Materials and Pharmaceutical Chemistry (Vietnam). We obtained 1ethyl-3-(3-dimethylaminopropyl)-carbodiimide (EDC) from Sigma Aldrich (United States). P. capsici, P. nicotianae, and $P$. colocasiae were isolated in the School of Agriculture and Forestry, Hue University (Vietnam).

2.2. Synthesis of DHPAC. The DHPAC was prepared from oligochitosan and 3,4-dihydroxyphenyl acetic acid in the presence of carbodiimide coupling reagent as shown in Scheme 1.

In summary, we added one gram of oligochitosan (6 mmol glucosamine) and $192 \mathrm{mg}$ of DHPA $(1.2 \mathrm{mmol})$ to 
$50 \mathrm{~mL}$ distilled water under stirring and then added $290 \mathrm{mg}$ of EDC $(1.4 \mathrm{mmol})$ to the mixture. The reaction mixture was stirred under nitrogen atmosphere at room temperature overnight and dialyzed with membrane with molecular weight cutoff of 6000-8000 afterward. After two days of dialysis, the polymer solution was lyophilized using a freezedrying machine to obtain DHPAC. The degree of DHPA substitution was estimated by ${ }^{1} \mathrm{H}$ NMR spectrum.

2.3. Preparation of Ag Core-DHPAC Shell NCs. Silver coreDHPAC shell NCs were prepared by mixing $50 \mathrm{~mL}$ DHPAC solution (1\%) containing around $1600 \mathrm{ppm}$ of DHPA (calculated according to ${ }^{1} \mathrm{H} \mathrm{NMR}$ ) and $50 \mathrm{~mL} \mathrm{AgNO}_{3}$ solution containing $800 \mathrm{ppm}$ of silver ion and then stirred for 30 minutes at room temperature. The Ag core-DHPAC shell NCs could be formed (as demonstrated in Figure 1) and characterized using UV-Vis, TEM, and XRD. For control sample, DHPA was the reducing agent without DHPAC. The experiment was conducted in the same manner with preparation of Ag core-shell NCs.

2.4. Characterization of Ag Core-DHPAC Shell NCs. UV-Vis absorption spectrum of the colloidal solutions was measured using Jasco V670. TEM images were collected using a JEM1400 instrument (JEM-1400, JEOL) which was operated at an accelerating voltage of $100 \mathrm{kV}$. Samples for TEM measurement were prepared by dropping AgNPs solution onto a carbon-coated copper grid. The colloidal solution-covered grids were allowed to dry for several hours. The XRD result was characterized using D8 advanced Bragg X-ray with $\mathrm{Cu}$ $\mathrm{K} \alpha$ radiation. For sample handling, glass slide was used as a substrate for measurement. Cleaned substrate was covered with the colloidal NCs and dried in air.

2.5. Studies on Growth Inhibition Ability of Plant-Pathogenic Fungi. The antifungal activity against pathogen was evaluated by using the in vitro plate dilution method. The colloidal NPs were mixed with $25 \mathrm{~mL}$ of melting potato dextrose agar (PDA) medium and then were poured into Petri dishes with final concentrations of 3,6 , and $9 \mathrm{ppm}$. The control dishes contained distilled water. The fungus was transferred equally onto the center point of the prepared Petri dishes and incubated at room temperature for six days. The growth inhibition of the fungus was evaluated by measuring the diameter of colony growth and calculated with the following formula: growth inhibition $(\%)=\left(\left(d_{1}-d_{2}\right) / d_{1}\right) \times 100$, where $d_{1}$ and $d_{2}$ are diameters of the colony of control and NCs-containing samples, respectively. All experiments were performed in triplicate.

\section{Results and Discussion}

3.1. Characterization of DHPAC. Structure of DHPAconjugated oligochitosan was well determined by the ${ }^{1} \mathrm{H}$ NMR spectrum. Figure 2 showed three aromatic protons of the conjugated DHPA with chemical shift $\left(\delta_{\mathrm{H}}\right)$ at 6.78 , 6.34 , and $6.73 \mathrm{ppm}$. Some typical glucosamine protons of

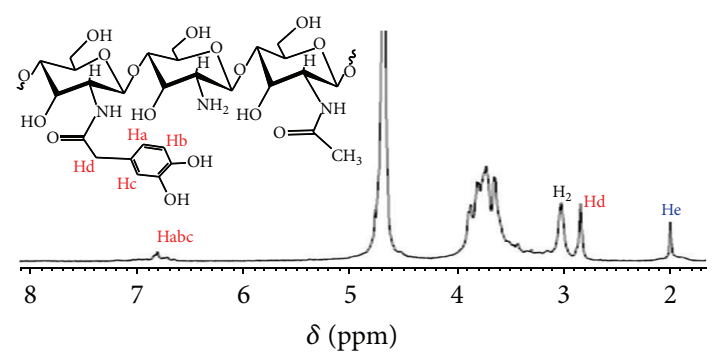

FIGURE 2: ${ }^{1} \mathrm{H}$ NMR spectra of chitosan-catechol derivative.

oligochitosan appear at $2 \mathrm{ppm}$ (acetyl protons), $3.02 \mathrm{ppm}$ ( $\mathrm{H}_{2}$ proton), and $3.5-4 \mathrm{ppm}\left(\mathrm{H}_{1-6}\right.$ protons). These results confirm that the DHPA was grafted to the chitosan effectively. According to integral values of DHPA aromatic protons and methyl protons of chitosan, the degree of DHPA substitution (DS) was calculated as 14 DHPA/100 glucosamine units.

3.2. Preparation of Ag Core-DHPAC Shell NCs. Preparation of AgNPs using natural extracts containing polyphenol compounds or catechol-functionalized polymers has recently been reported that is regarded as a green method [17-19]. In our study, DHPA moieties were utilized to reduce $\mathrm{Ag}^{+}$into a metallic form and then protected the produced AgNPs. After mixing DHPAC solution and $\mathrm{AgNO}_{3}$ solution, the color of the mixed solution immediately changed. After shaking for 15 minutes, UV-Vis absorbance of the solution performed two UV-Vis absorption peaks at wavelength ranging from 270 to $320 \mathrm{~nm}$ and 375 to $430 \mathrm{~nm}$ that was ascribed to absorbance of the DHPA moiety/oxidized DHPA product and surface plasmon resonance of AgNPs, respectively (as shown in Figure 3). The result indicated formation of the AgNPs.

Figure 4 showed XRD diffractogram of the Ag coreDHPAC shell NCs and the crystalline phase of metallic silver at $38.0,44.2,64.4,77.6$, and $81.6^{\circ}$. These peaks corresponded to the typical face centered cubic structure of Ag with miller indices at (111), (200), (220), (311), and (222), respectively $[4,20]$.

Figure 5 showed clearly that the core-shell NCs were formed in which Ag cores had the size distribution of $26 \pm$ $9 \mathrm{~nm}$ and polymer shell layer of $18 \pm 8 \mathrm{~nm}$ of thickness. These values were estimated by mean value of 50 selected nanoparticles. The experiment preparing only silver nanoparticle without shell had also been conducted to compare difference in morphology of AgNPs but the reaction mixture showed precipitation after $\mathrm{AgNO}_{3}$ and DHPA were added. This could be an aggregate of the formed AgNPs which was not well protected by polymer shell layer.

Formation of the polymer layer coating on the surface of the AgNPs could be explained as shown in Figure 6, in which some DHPA moieties on DHPAC reduced ionic silver to AgNPs and quinone moieties. Some DHPA moieties simultaneously adhered to the formed AgNPs via dopaminelike adhesion mechanism which was well proven in some previous report $[15,16,21,22]$. The quinone moieties from oxidized DHPAC easily reacted with amine groups of other 


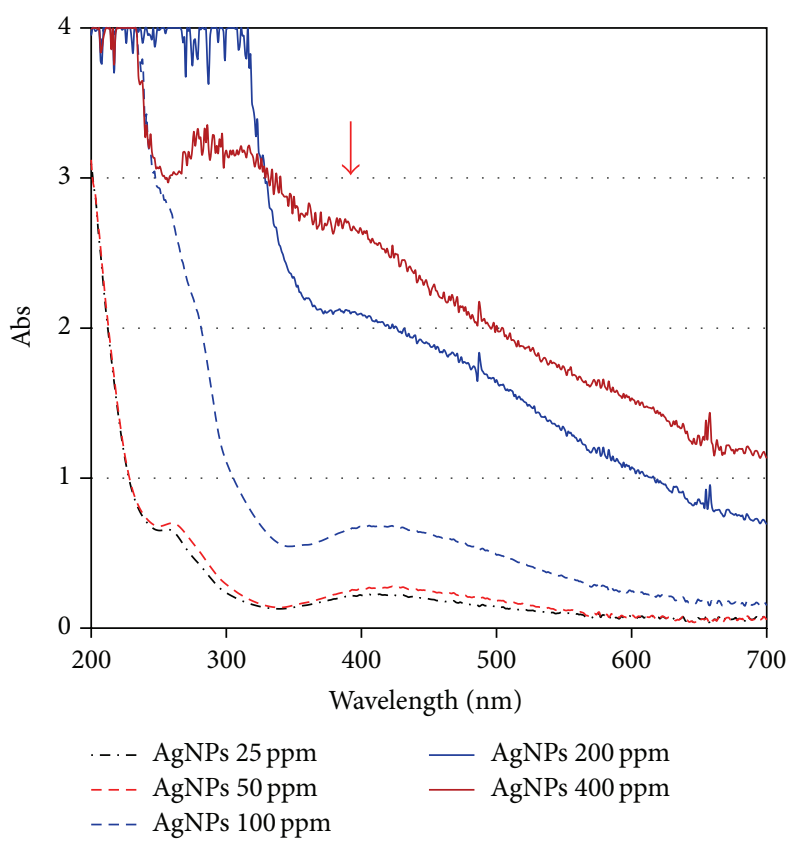

Figure 3: UV-Vis absorbance of Ag core-DHPAC shell NCs solution.

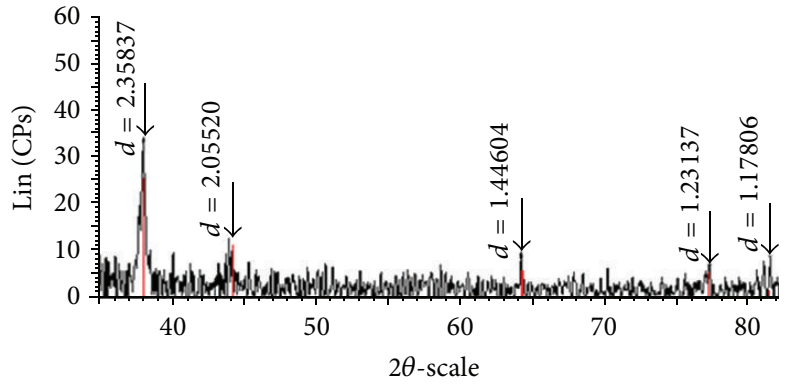

FIgURE 4: XRD pattern of the Ag core-DHPAC shell NCs.
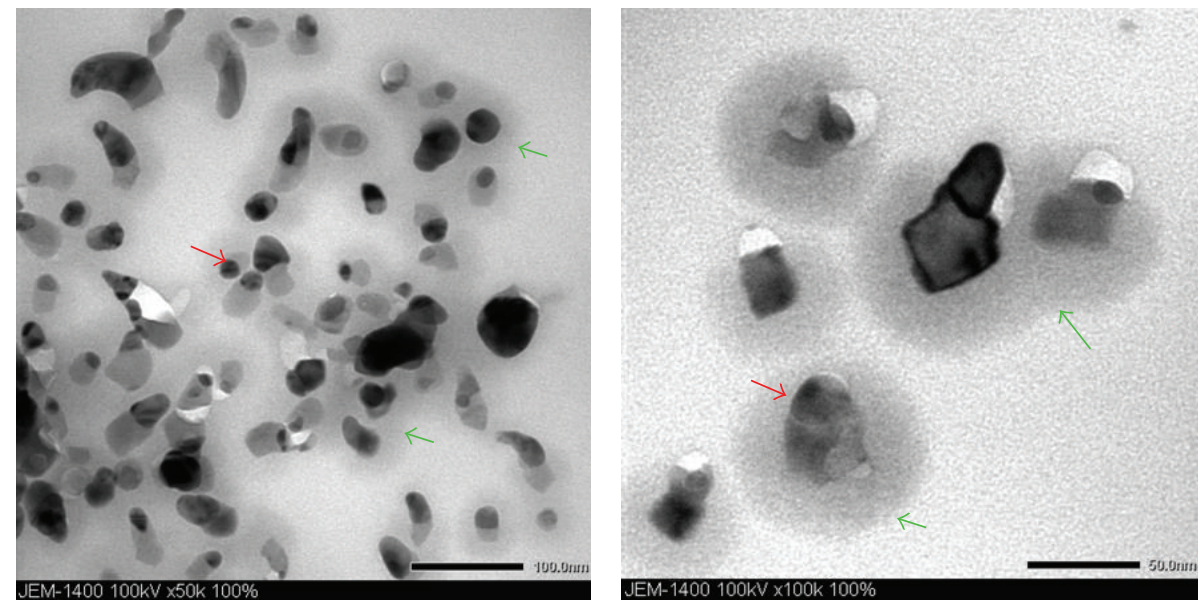

FIGURE 5: TEM images of the Ag core-DHPAC shell NCs: red and green arrows indicate Ag core and polymer shell layer, respectively. 

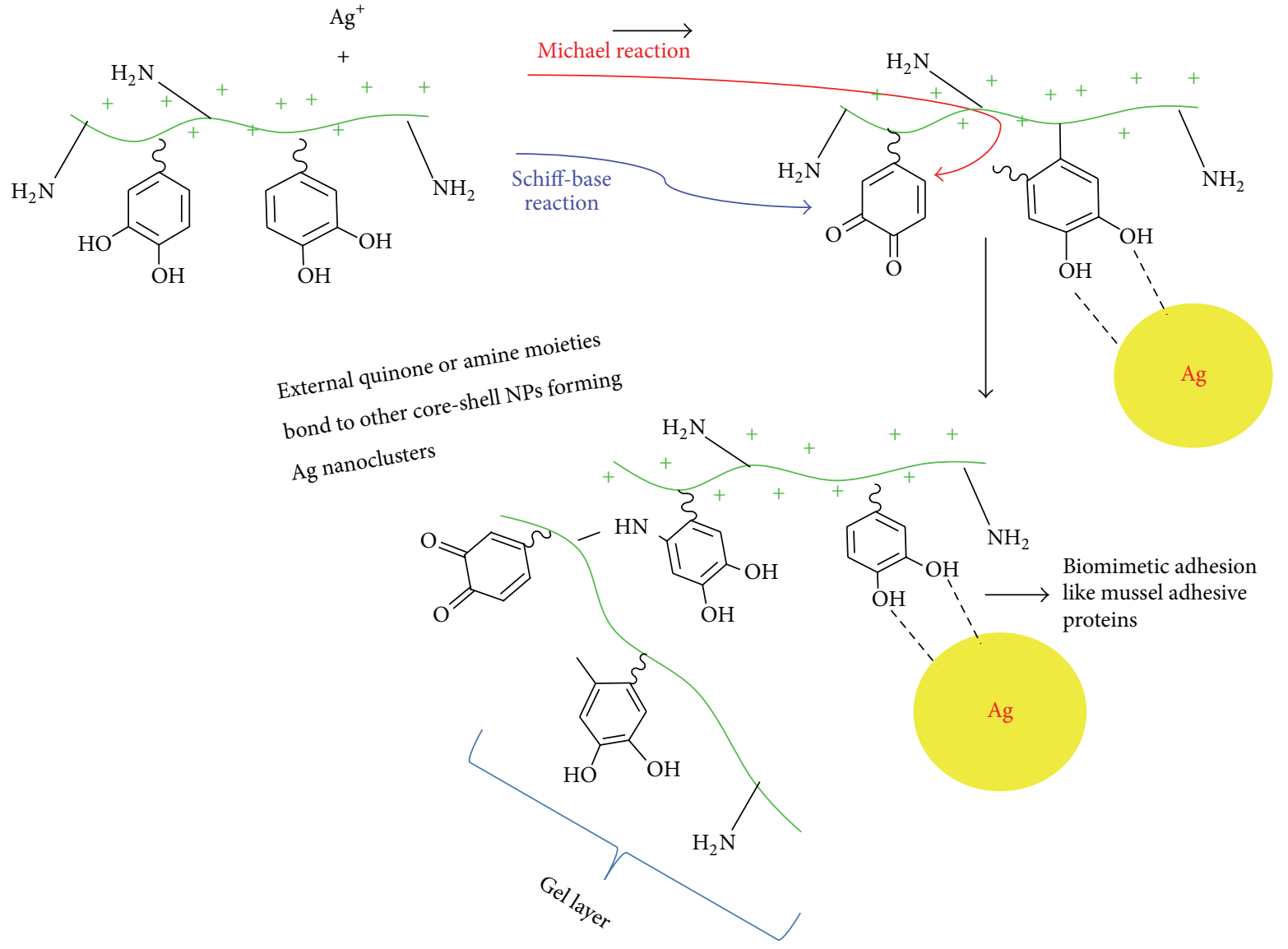

FIGURE 6: Core-shell AgNPs formation via biomimetic adhesion and chemical reactions.

DHPAC chains via Michael reaction between amine group of chitosan and double bond next to carbonyl of oxidized catechol or Schiff-base reaction between amine group of chitosan and carbonyl of oxidized catechol which resulted in formation of a polymeric hydrogel network. In the process, some functional groups on the core-shell AgNPs could bond together to form a nanocluster containing these AgNPs.

\subsection{Antibacterial Activity of Ag Core-DHPAC Shell NCs. The} Ag core-DHPAC shell NCs exhibited strong growth inhibition of plant-pathogenic fungi such as $P$. capsici, $P$. nicotianae, and $P$. colocasiae. Figure 7 showed results obtained from antifungal experiments of the AgNCs colloidal solutions. There was strong growth of these fungi in control sample without AgNCs. A high colony diameter of the fungi was recorded. In the presence of small amount of AgNCs (3 ppm), growth of the fungi was significantly decreased.

Figure 7 showed growth inhibition of these fungi, in which $P$. capsici fungus was inhibited at $80 \%$ in the presence of 9 ppm AgNCs. Growth inhibition of P. nicotianae and P. colocasiae was approximated. Fifty percent growth inhibition of these fungi was recorded at six ppm of AgNCs approximately, which was a low concentration compared to effective dose (ED50) of inhibition for plant-pathogenic Corticium salmonicolor at $27.2 \mathrm{ppm}$ of AgNPs [23]. In additional experiment, antifungal activity of oligochitosan has also been conducted. In the same concentration with DHPAC used in preparation of AgNCs, the chitosan was diluted in the same manner to the highest concentration at $45 \mathrm{ppm}$. In the concentration, chitosan did not show antifungal activity. It was in agreement with a previous report in which oligochitosan polymer solution showed antibacterial activity at high concentration (100 ppm) [22].

Different behaviors might be due to differences in organizations, structures, and functions of these plant-pathogenic fungi or due to different interaction of the core-shell AgNPs and oligochitosan with the fungi (Figure 8).

For the Ag core-DHPAC shell NCs, chitosan-based shell layer might improve antifungal activity of the AgNCs because of cationic polymer possessing antimicrobial or antipathogenic activity. Xu et al. reported that oligochitosan exhibited the highest inhibitory growth with $P$. capsici compared to other phytopathogens. Fifty percent inhibitory growth of $P$. capsici was reported at $100 \mathrm{ppm}$ of oligochitosan [24]. Although the activity was much lower than that of AgNPs, the polymer layer could act as an active targeting site and result in increasing interaction of the cationic chitosan shell layer on Ag core in the NCs and phospholipid layer on bacterial membrane via electrostatic interaction. This brought the Ag core-DHPAC shell NCs to the surface of the microbes and sustainedly released $\mathrm{Ag}^{+}$ions could kill the 


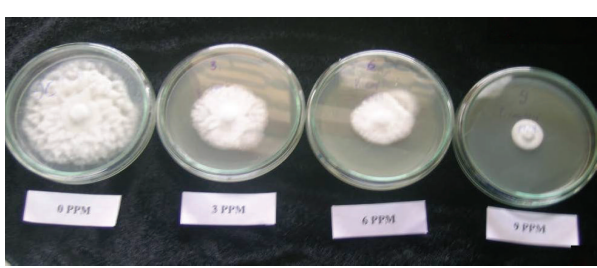

(a)

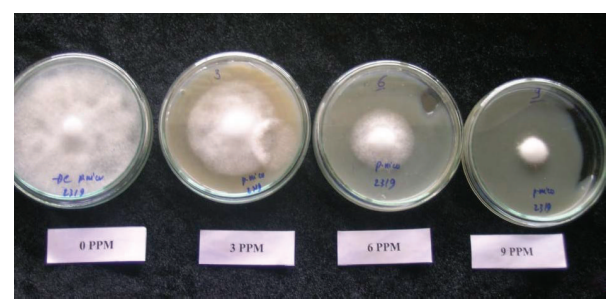

(b)

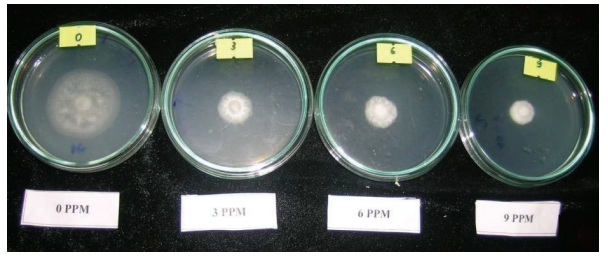

(c)

FIgURE 7: Growth of the fungi in diffrent PDA media with and without AgNCs: P. capsici (a), P. nicotianae (b), and P. colocasiae (c).



(a)

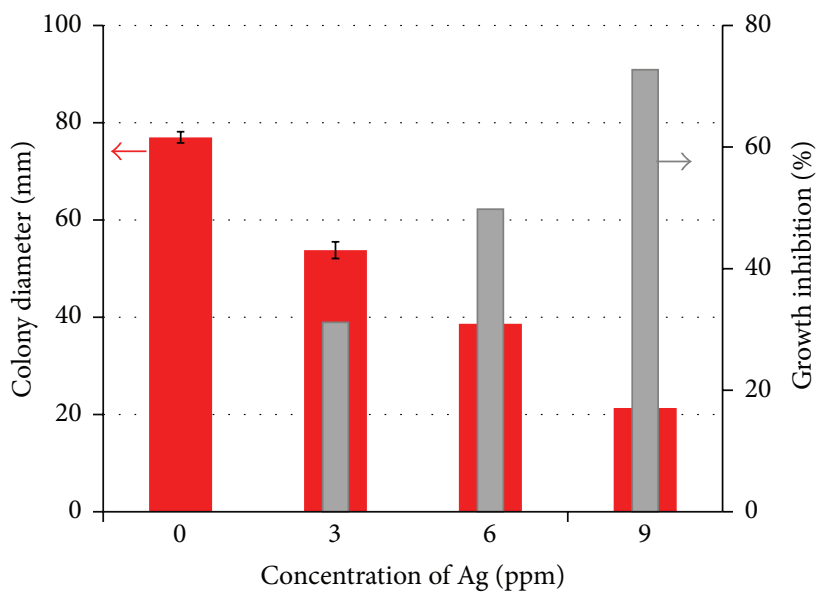

(b)

FIGURE 8: Growth inhibition of Ag core-DHPAC shell NCs on the plant-pathogenic fungi: P. capsici (a) and P. nicotianae (b).

fungi. Slade and Pegg reported that several kinds of Phytophthora fungi were killed by $\mathrm{Ag}^{+}$in the range 46-460 nM (5-50 ppb) [25]. Sustained release of $\mathrm{Ag}^{+}$ions from the Ag core-DHPAC shell NCs could be significant to inhibit growth of the plant-pathogenic fungi and protect plants from Phytophthora fungi.

\section{Conclusion}

In this work, silver core-chitosan shell nanoclusters were prepared via chemical reduction using chitosan derivative as a reducing and protecting agent. Low concentration of the nanoparticles exhibited a powerful activity against plant pathogens, such as $P$. capsici, $P$. nicotianae, and $P$. colocasiae. Positive results show the potential application as an inexpensive ecofungicide for agriculture.

\section{Conflict of Interests}

The authors declare that there is no conflict of interests regarding the publication of this paper.

\section{Acknowledgment}

This research was supported by Vietnam National Foundation for Science and Technology Development (NAFOSTED) under Grant no. 104.04-2011.49.

\section{References}

[1] M. de, P. S. Ghosh, and V. M. Rotello, "Applications of nanoparticles in biology," Advanced Materials, vol. 20, no. 22, pp. 42254241, 2008. 
[2] M. Abhilash, "Potential applications of nanoparticles," International Journal of Pharma and Bio Sciences, vol. 1, no. 1, pp. 1-12, 2010.

[3] M. Sharon, A. K. Choudhary, and R. Kumar, "Nanotechnology in agricultural disease and food safety," Journal of Phytomedicine, vol. 2, no. 4, pp. 83-92, 2010.

[4] J. P. Ruparelia, A. K. Chatterjee, S. P. Duttagupta, and S. Mukherji, "Strain specificity in antimicrobial activity of silver and copper nanoparticles," Acta Biomaterialia, vol. 4, no. 3, pp. 707-716, 2008.

[5] L. Rastogi and J. Arunachalam, "Synthesis and characterization of bovine serum albumin-copper nanocomposites for antibacterial applications," Colloids and Surfaces B: Biointerfaces, vol. 108, pp. 134-141, 2013.

[6] K. Adavallan and N. Krishnakumar, "Mulberry leaf extract mediated synthesis of gold nanoparticles and its anti-bacterial activity against human pathogens," Advances in Natural Sciences: Nanoscience and Nanotechnology, vol. 5, no. 2, Article ID 025018, 2014.

[7] V. D. Cao, P. P. Nguyen, V. Q. Khuong et al., "Ultrafine copper nanoparticles exhibiting a powerful antifungal/killing activity against Corticium salmonicolor," Bulletin of the Korean Chemical Society, vol. 35, no. 9, pp. 2645-2648, 2014.

[8] X. Wang, F. Cheng, J. Gao, and L. Wang, "Antibacterial wound dressing from chitosan/polyethylene oxide nanofibers mats embedded with silver nanoparticles," Journal of Biomaterials Applications, 2014.

[9] Q. H. Tran, V. Q. Nguyen, and A.-T. Le, “ Silver nanoparticles: synthesis, properties, toxicology, applications and perspectives," Advances in Natural Sciences: Nanoscience and Nanotechnology, vol. 4, Article ID 033001, 2013.

[10] R. M. Tripathi, R. K. Gupta, A. Shrivastav, M. P. Singh, B. R. Shrivastav, and P. Singh, "Trichoderma koningii assisted biogenic synthesis of silver nanoparticles and evaluation of their antibacterial activity," Advances in Natural Sciences: Nanoscience and Nanotechnology, vol. 4, no. 3, Article ID 035005, 2013.

[11] H.-J. Park, S. H. Kim, H. J. Kim, and S.-H. Choi, "A new composition of nanosized silica-silver for control of various plant diseases," Plant Pathology Journal, vol. 22, no. 3, pp. 295-302, 2006.

[12] T. S. Cu, V. D. Cao, C. K. Nguyen, and N. Q. Tran, "Preparation of silver core-chitosan shell nanoparticles using catecholfunctionalized chitosan and antibacterial studies," Macromolecular Research, vol. 22, no. 4, pp. 418-423, 2014.

[13] N. V. Truong, L. W. Burgess, and E. C. Y. Liew, "Greenhouse and field evaluations of potassium phosphonate: the control of Phytophthora foot rot of black pepper in Vietnam," Archives of Phytopathology and Plant Protection, vol. 45, no. 6, pp. 724-739, 2012.

[14] N. V. Truong, L. W. Burgess, and E. C. Y. Liew, "Cross-infectivity and genetic variation of Phytophthora capsici isolates from chilli and black pepper in Vietnam," Australasian Plant Pathology, vol. 41, no. 4, pp. 439-447, 2012.

[15] H. Lee, S. M. Dellatore, W. M. Miller, and P. B. Messersmith, "Mussel-inspired surface chemistry for multifunctional coatings," Science, vol. 318, no. 5849, pp. 426-430, 2007.

[16] J. H. Waite, "Surface chemistry: mussel power," Nature Materials, vol. 7, pp. 7-9, 2008.

[17] E. Rodríguez-León, R. Iñiguez-Palomares, R. E. Navarro et al., "Synthesis of silver nanoparticles using reducing agents obtained from natural sources (Rumex hymenosepalus extracts)," Nanoscale Research Letters, vol. 8, no. 1, article 318, 2013.

[18] F. Cataldo, "Green synthesis of silver nanoparticles by the action of black or green tea infusions on silver ions," European Chemical Bulletin, vol. 3, no. 3, pp. 280-289, 2014.

[19] K. C. L. Black, Z. Liu, and P. B. Messersmith, "Catechol redox induced formation of metal core-polymer shell nanoparticles," Chemistry of Materials, vol. 23, no. 5, pp. 1130-1135, 2011.

[20] S. Zaki, M. Etarahony, M. Elkady, and D. Abd-El-Haleem, "The use of bioflocculant and bioflocculant-producing Bacillus mojavensis strain 32A to synthesize silver nanoparticles," Journal of Nanomaterials, vol. 2014, Article ID 431089, 7 pages, 2014.

[21] Y. Lee, H. Lee, P. B. Messersmith, and T. G. Park, "A bioinspired polymeric template for $1 \mathrm{D}$ assembly of metallic nanoparticles, semiconductor quantum dots, and magnetic nanoparticles," Macromolecular Rapid Communications, vol. 31, no. 24, pp. 2109-2114, 2010.

[22] H. Lee, N. F. Scherer, and P. B. Messersmith, "Single-molecule mechanics of mussel adhesion," Proceedings of the National Academy of Sciences of the United States of America, vol. 103, no. 35, pp. 12999-13003, 2006.

[23] D. van Phu, V. T. K. Lang, N. T. K. Lan et al., "Synthesis and antimicrobial effects of colloidal silver nanoparticles in chitosan by $\gamma$-irradiation," Journal of Experimental Nanoscience, vol. 5, no. 2, pp. 169-179, 2010.

[24] J. Xu, X. Zhao, X. Han, and Y. Du, "Antifungal activity of oligochitosan against Phytophthora capsici and other plant pathogenic fungi in vitro," Pesticide Biochemistry and Physiology, vol. 87, no. 3, pp. 220-228, 2007.

[25] S. J. Slade and G. F. Pegg, "The effect of silver and other metal ions on the in vitro growth of root-rotting Phytophthora and other fungal species," Annals of Applied Biology, vol. 122, no. 2, pp. 233-251, 1993. 

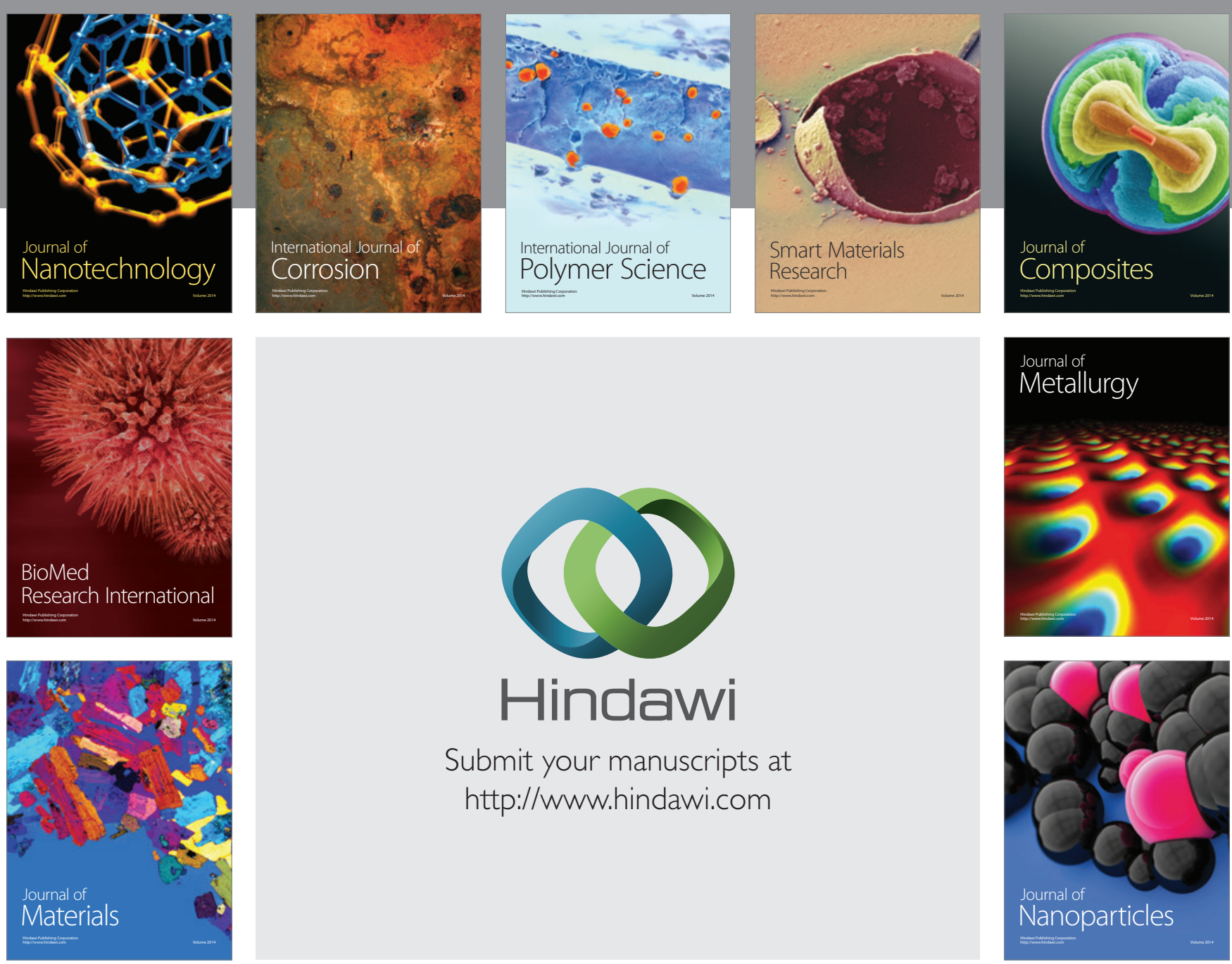

Submit your manuscripts at http://www.hindawi.com
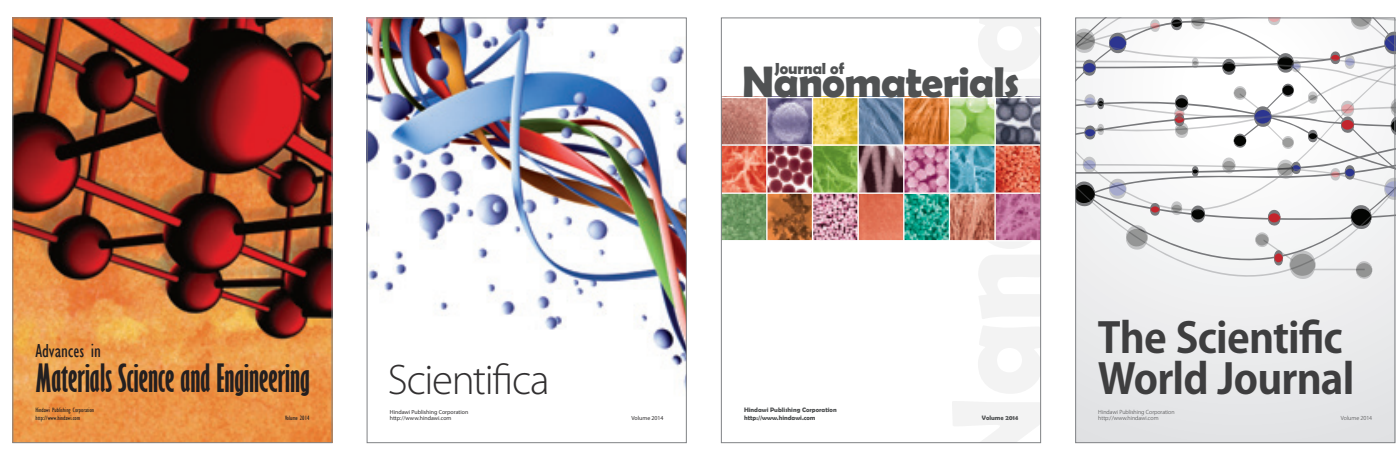

\section{The Scientific World Journal}
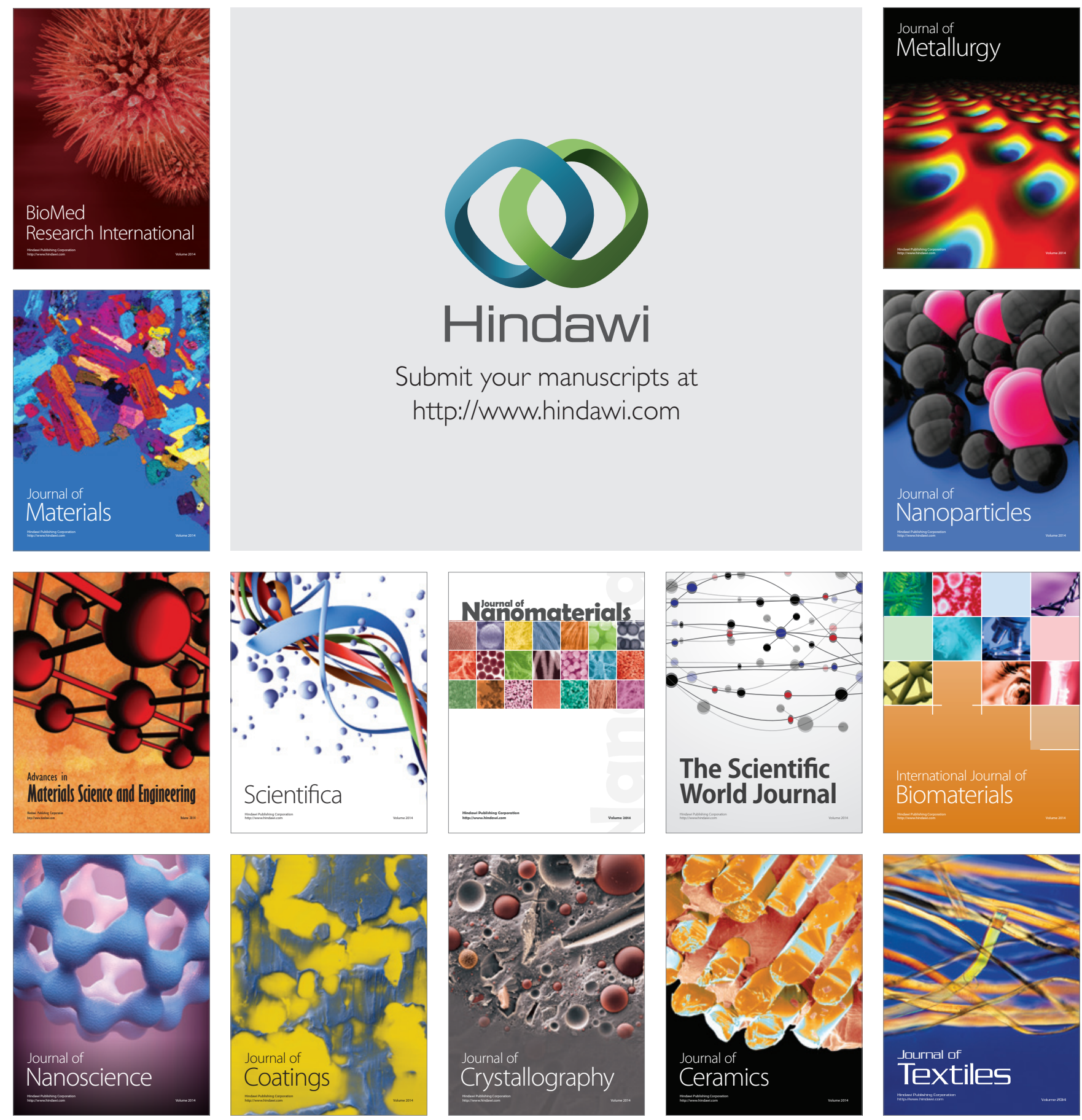